\title{
The Applied Research of the Network Coding Technology in the Transmission Mode of Multi-point to Single point Wireless Signal
}

\author{
LI Yue ${ }^{1, a *}$, PENG Zuhua ${ }^{1, a}$ \\ 1 State Grid Xinyang Power Supply Company, Henan, Xinyang \\ a55609170@qq.com
}

\begin{abstract}
Keywords: Network Coding; Wireless Network; Application Mode.
\end{abstract}
Abstract. Nowadays, the biggest problem that has influenced the using effect of wireless networks is that the transmission signals are easy to be affected by the external factors as well as the hardware equipments which are easy to be damaged, which lead to the phenomena like bad performance of transmission signals, the limitation of network resource and even the break-off of the network. Network coding technology is not only able to improve greatly the reliability and the safety of the wireless network channels but also it is capable to improve the throughput of network and the utilization efficiency of resources, which shows up the higher value of utilization. Based on this theory, taking the transmission mode of multi-point to single point wireless signal, one of the most principal and the most fundamental communication methods of the wireless mesh network as the object of study, this paper analyzes systematically the applied feasibility situation of network coding technology in this mode, and discusses systematically its application method and the application effect situation to offer a brand new perspective to further upgrade the wireless transmission technology from this.

\section{Introduction}

For the past few years, how to avoid the failure of network links or network nodes in the process of wireless network transmission is an important issue pondered by many experts and scholars. Thereinto, the protection mechanism based on network coding, namely $1+\mathrm{N}$ protection mechanism, is a new type of network protection mechanism based on the advantages and disadvantages assimilated as well as used for reference by active protection mechanism $(1+1$ protection mechanism) and passive protection mechanism (1: $\mathrm{N}$ protection mechanism). When there are $\mathrm{N}$ relay nodes in the wireless network, the system sets up $1+\mathrm{N}$ paths leading to the goal nodes for each source node, encodes the transfer data into $1+\mathrm{N}$ encoded packets through $1+\mathrm{N}$ mutually disjoint paths to the goal nodes; if the loss of data comes out, with the help of little network resources the system can restore the lost data. This mode gives good consideration to the efficiency and the timeliness of the network protection and it is relatively ideal active network fault-tolerant protection mechanism. Based on this theory, this paper presents a new method and measure that network coding technology realizes the $1+\mathrm{N}$ protection mechanism in wireless mesh network, especially it can apply to the general network topology; from this the waste of network resources is avoided, and the utilization efficiency of network resources is greatly improved

\section{Explorations and analysis of the feasibility of the $1+\mathrm{N}$ protection mechanism applied to the wireless mesh network}

Multi-point to single point wireless signal transmission mode is one of the most principal and the most fundamental communication methods.

Supposing that the general network topology of wireless mesh network is G, Us as a group of sending source nodes, Ls as a group of routing nodes, $\mathrm{P}$ as the mutually disjoint path from routing nodes to gateway, and the distribution of a group of mutually disjoint paths for each routing node as $\mathrm{Pi}=\{\mathrm{Pi}, 1, \mathrm{Pi}, 2, \cdots \cdots, \mathrm{Pil}, \mathrm{Pil}\}$, among them for $\mathrm{Vi}, \mathrm{j}, \mathrm{Pi}, \mathrm{j} \epsilon \mathrm{P}$, and when $\mathrm{Vi} \neq \mathrm{j}$, there is $\mathrm{PinPj}=\Phi$, on this basis, we can adopt these steps to build a auxiliary chart $G$ ': 
First of all, the auxiliary chart $G$ ' still includes the 0 node which keeps the corresponding relation with a specific gateway node in the original network diagram G. Secondly, in the auxiliary chart G' there is also a li node and every router node li which doesn't belong to Ls in the original network diagram $\mathrm{G}$, the information channel quantities of the nodes in the original network diagram and in the auxiliary chart to the router nodes are coincident. Thirdly, if each router node in the original network diagram is distributed with / $\mathrm{Pi}$ / mutually disjoint paths connected to the gateway, and in the auxiliary chart $G^{\prime}$ there are the correspondent paths, so this makes every lij node to possess a path Pij leading to the gateway and Internet. Fourthly, there is a source node uk in the auxiliary chart G' and there is each source node ukEUs in the original network diagram G, so these form the set U's of source nodes. The links in the auxiliary chart $G^{\prime}$ keep the relation of one-to-one correspondence with those in the network diagram $\mathrm{G}$.

It can be seen that there is at most only one path which gets to the node 0 among the source nodes that connect to the router nodes in the auxiliary chart G', so these meet the former assumed condition, namely there is only one connected path between any one of the router nodes and the gateway node; based on this, we can adopt the network coding technology to design a $1+\mathrm{N}$ protection mechanism for the entire network system.

Firstly, the encoded packets with the specific amount in the auxiliary chart G' transmit from the node uk to li, j, so the encoded packets with the same amount in the network topological graph transmit from uk to li. Secondly, the amount of the encoded packets transmitted on the path $\mathrm{Pi}, \mathrm{j}$ in the network topological graph keeps the same with those of the data packets transmitted on the path $\mathrm{Pi}, \mathrm{j}$ in the auxiliary chart $\mathrm{G}^{\prime}$. The values of $\mathrm{k}, \mathrm{i}, \mathrm{j}$ are gained randomly.

In the auxiliary chart $G^{\prime}$, it is principally the intermediate node or the routing node $\mathrm{li}, \mathrm{j}$ that is in charge of encoding activities, and the received data packets whose encoding in progress keep the same with the data packets received and corresponding to the nodes in the network topological graph G. Based on this, if the routing nodes in the network topological graph $\mathrm{G}$ can encode the data packets, the routing nodes in the auxiliary chart $G^{\prime}$ can execute the linear operations for the same data packets and transmits the data packets after the encoding on the path $\mathrm{Pi}$, j. According to the action mode of $1+\mathrm{N}$ protection mechanism in the beginning part of this chapter, because this mechanism can offer the protection to the entire network, when a source node goes wrong, the goal nodes can obtain $\mathrm{N}$ same encoded data packets by other $\mathrm{N}$ mutually disjoint paths. This network coding technology based on $1+\mathrm{N}$ protection mechanism can really and greatly improve the reliability of the information channels in the auxiliary chart G'. From another point of view, in the auxiliary chart G', it can be connected by $\mathrm{K}+1$ paths between any $\mathrm{K}$ nodes and the routing nodes, so there is indeed a network coding project that can ensure the goal nodes to receive the encoded data packets when a path from the source nodes goes wrong. This fully proves that the general network topology can adopt $1+\mathrm{N}$ protection mechanism to improve the reliability of the information channels under the circumstance of the assumptions.

\section{Explorations and analysis of the methods in the $1+\mathrm{N}$ protection mechanism applied to the wireless mesh network}

\section{The algorithm design of the $1+\mathrm{N}$ protection mechanism}

Above all, some scholars fully prove at present that the polynomial time in the auxiliary chart G' can verify the necessary and sufficient condition in the theorem 1 proposed. The book The Design of Wireless Network Coding gives a strict definition that the topological graph network means only one path connected to the wireless gateway. Besides, it can be seen from the analysis of the theorem 1 that if we can adopt the polynomial time to verify the auxiliary chart $G^{\prime}$ can meet the conditions proposed by theorem 1 , as a result, we can know it is viable that adopting the $1+\mathrm{N}$ protection mechanism based on network coding technology provides guarantees for the feasibility of the information channels of the entire network system within the polynomial time. It also can be seen that if we can use the polynomial time to verify the theorem 1 , the theorem 2 can also get the valid proof. 


\section{The project design of the $1+\mathrm{N}$ protection mechanism}

We can design a set of $1+\mathrm{N}$ protection mechanisms for the general network topology to provide the guarantee for the reliability of the information channels of the entire system. Suppose that $d^{\prime} i, j$ are the encoded data packets transmitting on the path pi,j of the auxiliary chart $G$ ', so the encoded data packets in the original network diagram $G$ corresponding to them are di,j. According to this, we can use the network coding technology and follow the steps below to design a set of the $1+\mathrm{N}$ protection mechanism for the whole system.

In the process of build the auxiliary chart G', we know that the relation of one-to-one correspondence exits between from a node to the edge of the node in the auxiliary chart $G^{\prime}$ and the original network diagram $G$, as a result, if the source node uk in the auxiliary chart $G^{\prime}$ makes the original data to transmit to li, $\mathrm{j}$; the original node $\mathrm{uk}$ in the original network diagram $\mathrm{G}$ transmits the original data packets with it to the corresponding node li; because all the nodes in the auxiliary chart $\mathrm{G}^{\prime}$ are derived from the routing nodes $\mathrm{li}$ in the original network diagram $\mathrm{G}$, as a consequence, the nodes li on the original network diagram $\mathrm{G}$ can produce the encoded data packets proceeding the transmission on the routing nodes li that can be connected with them.

From this, in the coding project of the $1+\mathrm{N}$ protection mechanism designed, the encoded packets from the rendezvous points or the gateway in the original network diagram $G$ and the auxiliary chart $\mathrm{G}^{\prime}$ is not only one-to-one corresponding and synchronous, and its contents are totally the same; as a consequence, the coding project of the $1+\mathrm{N}$ protection mechanism designed can produce the good protective effects for the information channels of the entire system in the general topological network in the wireless mesh network.

\section{Analysis for the effects of the $1+\mathrm{N}$ protection mechanism applied to the wireless mesh network}

To verify the real effect of the $1+\mathrm{N}$ protection mechanism applied to the wireless mesh network, we need to create at random a general network topology. First of all, we need to create randomly Us systematic source nodes, Ls routing nodes connected to them and a rendezvous point (gateway node); then we make it possible for all the source nodes to connect to k routing nodes in the Ls, and make the each routing nodes in the Ls to possess $\mathrm{p}$ mutually disjoint information channels to connect to the rendezvous point. Aiming at the four parameters above, the system creates randomly 40 totally different general network topologies; then we use the $1+\mathrm{N}$ protection mechanism mentioned in the preceding part of the paper and the other $1+\mathrm{N}$ protection mechanisms proposed by the scholars at present to protect and test each network topology, we analyze the results of the tests by the comparison, and we can verify the actual efficiency by the $1+\mathrm{N}$ protection mechanism proposed for the network system.

First of all, under the circumstances when the amount of users whose data need to be transmitted or the amount of source nodes is increasing continuously, we compare the data throughputs of the two projects. Specifically, the system fixes 14 routing nodes, every routing node adopts two mutually disjoint paths connected to the congruent point, the source nodes can randomly choose 4 routing nodes to transmit data packets, and the number increases gradually from 4 to 40 , we suppose that the sending speed is $10 \mathrm{Mbps}$.

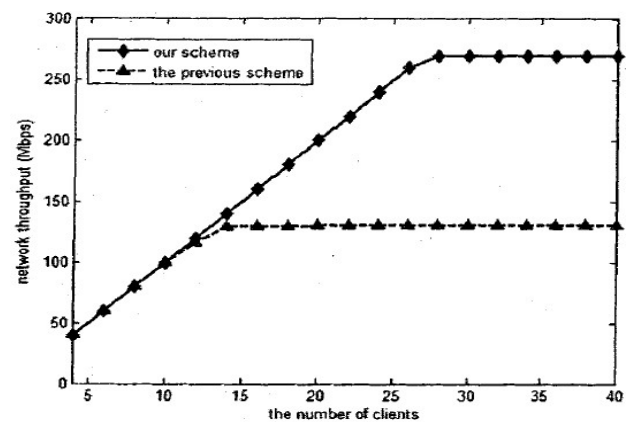

Fig.1

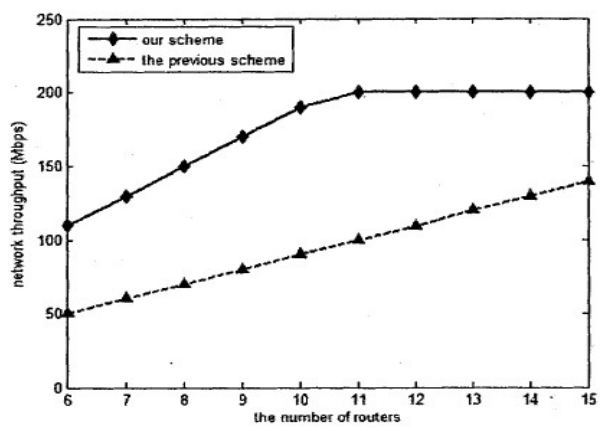

Fig. 2

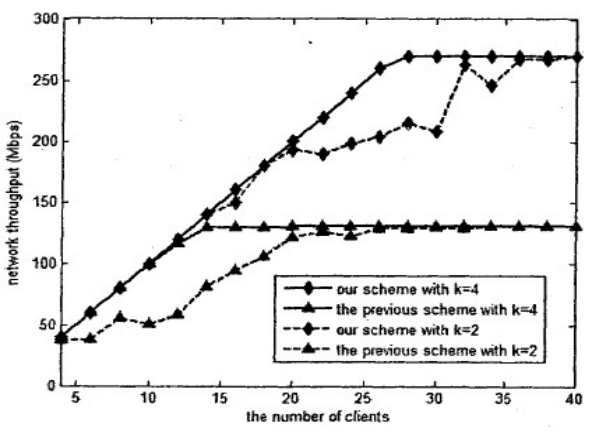

Fig. 3 
The ordinate in the graph above represents the continuous increasing of the amount of source nodes, the abscissa represents the situation of the throughput in the system, and each value in the graph is the average value produced by the 40 random topological networks under different protection mechanisms. We can see that the throughput borne by the $1+\mathrm{N}$ protection mechanism designed and based on the network coding technology is much higher than those of the other sort of $1+\mathrm{N}$ protection mechanism. The reason is that although ordinary $1+\mathrm{N}$ protection mechanism has several paths connected to the routing nodes and the congruent point to transmit encoded data packets, there is only one path to transmit them, which results in the immense waste of network resources and the limitation of the network capacity. But if we adopt the $1+\mathrm{N}$ protection mechanism designed, we can fully make use of each path connected the routing nodes and the congruent point to transmit the encoded data packets, so we can greatly improve the throughput of the system as well as the utilization efficiency of network resources. From another point of view, it proves that the $1+\mathrm{N}$ protection mechanism proposed can protect more source nodes at the same time to carry out the data transmitting activities.

Secondly, under the circumstance of the increasing amount of routing nodes connected to the specific source nodes, we test the throughput of the $1+\mathrm{N}$ protection mechanism proposed. Supposing that the number of the source nodes in the network is 20 , and sending speed is $10 \mathrm{Mbps}$, then the quantity of system flow is $200 \mathrm{Mbps}$, there are two mutually disjoint paths connecting each routing node and the congruent point, and the source nodes can randomly choose 2 routing nodes to transmit the data. As what is shown in Graph 3-2, when the number of the routing nodes increases from 6 to 15, the changing situation of 40 throughputs of network topological system is:

In the Graph 2, the abscissa represents the number of the routing nodes, the ordinate represents the situation of the throughput, and every point in the graph is the average value of 40 ordinary topological networks. After careful analysis, we found that the $1+\mathrm{N}$ protection mechanism with the continuous increasing of the routing nodes can make the system to obtain the higher throughput. The reason is that when one routing node increases in the ordinary $1+\mathrm{N}$ protection mechanism, it simply offer a extra path resource to each source node; but when each routing node increases, the $1+\mathrm{N}$ protection mechanism designed offer two extra path resources to every source node, so this makes the throughput of the entire system higher than those under the ordinary $1+\mathrm{N}$ protection mechanism.

Thirdly, when there are two or four mutually disjoint paths connecting the routing nodes and the congruent point, with the process of the unceasing increasing of the user's demands or the number of the source nodes, this is the situation of system throughputs brought by the different protection mechanisms. Suppose that there are 14 routing nodes in the system, they possess 2 paths connecting with the congruent nodes, and every source node can randomly choose $\mathrm{k}$ paths to send source data packets to the routing nodes, speed is $10 \mathrm{Mbps}$, when the number of the source nodes increases from 4 to 40 , as what is shown in the Graph $3-3$, the comparison of the system throughputs brought by various kinds of protection mechanisms:

In the Graph 3, the abscissa represents the number of the source nodes, and the ordinate represents the system throughputs. From the analysis, when the value of $\mathrm{k}$ is certain, with the unceasing increasing of source nodes amount, the network throughput of the $1+\mathrm{N}$ protection mechanism designed also shows up the trend of fast rise. The reason why these two curves tend to be stable is that because the number of the routing nodes and the number of those paths connected to the congruent point are both fixed, the change of the network topology does not influence the transmitting ability of the system; and when source nodes increase to a certain level, the network load reaches to its limit, and it cannot rise the throughput any more. This manifests that the $1+\mathrm{N}$ protection mechanism designed in the general topological network has the obvious advantage in rising the system throughput. 


\section{Conclusion}

Through the test by the experiments, it is feasible and workable for adopting the network coding technology to solve the facing problem in the operation of multi-point to single point wireless signal transmission mode, and it has a certain value of popularization. In the long run, the applied research of the network coding technology in the wireless network also needs to pay attention to the problem like the plenty of failures under multi-point to single point wireless signal transmission mode in the wireless mesh network, etc, to improve further the technological level of the network coding technology in the wireless network.

\section{References}

[1] Song Xiaoquan, The Research Based on the Feasibility of Wireless Network of Random Network Coding, Journal of Shenzhen University, (1) 2014.

[2]Wang Bin, The Wireless Network Coding Mechanism of Efficiency Perception [J], Journal of Electronics \& Information Technology, (1) 2012

\section{Reference to a book:}

[3] Wu Zhanji, Wireless Network Coding__Theories and Applications, Beijing: Tsinghua University Press, July 2014. 